\title{
A Case of Vascular Malformation of the Neck
}

\author{
M. Senthilvelan • C. Sarath Chandran • \\ C. S. Subramanian • M. Prema • P. Umapathi
}

Received: 25 April 2014 / Accepted: 13 July 2014 / Published online: 23 July 2014

(C) Association of Surgeons of India 2014

\begin{abstract}
Vascular malformations are rare congenital vascular anomalies composed of inappropriately connected vasculature. They are usually present at birth, are progressive, infiltrative and require intervention. Vascular malformations need to be differentiated from haemangiomas which are congenital vascular neoplasms. We present a case of vascular malformation in a 6-year old child who presented with a progressive swelling in the neck and was treated by surgical excision. This case is being presented because of its peculiar clinical presentation.
\end{abstract}

Keywords Vascular anomaly · Vascular malformation . Haemangioma $\cdot$ Neck swelling

\section{Introduction}

Vascular anomalies are a heterogeneous group of congenital blood vessel disorders more typically referred to as birthmarks. They are broadly classified into haemangiomas and vascular malformations based on clinical presentation, histopathologic features and biological behaviour [1]. We present a case of vascular malformation in a 6-year old child who presented with a progressive swelling in the deep intermuscular compartment of the neck without skin discolouration. This case is presented because of its peculiar clinical presentation.

M. Senthilvelan $(\varangle) \cdot$ C. Sarath Chandran · C. S. Subramanian • M. Prema $\cdot$ P. Umapathi

Department of General Surgery, Raja Muthiah Medical College, Annamalai University, Chidambaram, Tamil Nadu, India

e-mail:drmsv67@gmail.com

\section{Case Record}

A 6-year old male child presented in our outpatient department with history of swelling in the right side of the neck since birth. The swelling was painless initially but was associated with recent increase in size and pain for the past 2 weeks. The swelling did not cause any pressure symptoms. There was no history of cough or loss of weight or other constitutional symptoms. On examination, the child had a $7 \times 6 \mathrm{~cm}$ swelling in the right upper and lateral aspect of the neck involving carotid and posterior triangles of the neck. The swelling was variable in consistency with solid and cystic areas and nontransilluminant. The swelling moved with deglutition. The swelling was not compressible but transmitted pulsation was felt over the swelling. Skin over the swelling was normal. Examination of oral cavity was normal. A provisional diagnosis of vascular malformation possibly lymphatic malformation (cystic hygroma) was made. Differential diagnosis considered was haemangioma, branchial cyst and cold abscess of cervical lymph nodes. Ultrasonogram of the neck showed a multiloculated cystic lesion of size $3.7 \times 2.2 \times 1.7 \mathrm{~cm}$ in the anterior and posterior triangles of the neck deep to sternocleidomastoid with peripheral vascular uptake. A computed tomography showed a well encapsulated, mixed echogenic mass with multiple septations, solid and cystic areas of size $4 \times 4.3 \times 18.5 \mathrm{~cm}$ in the anterior deep cervical compartment. Trachea, carotid artery and jugular vein were displaced, and thyroid lobe was found compressed. No infiltration into vessels was found (Fig. 1). Patient was taken up for surgery under general anaesthesia. During surgery, a welldefined multiloculated cystic and vascular swelling were found adherent to surrounding muscles and carotid sheath (Fig. 2). Medial part of the swelling was found adherent to right lobe of thyroid which explains movement of the swelling with deglutition. The swelling was excised in toto preserving strap muscles, sternocleidomastoid, carotid sheath and thyroid 


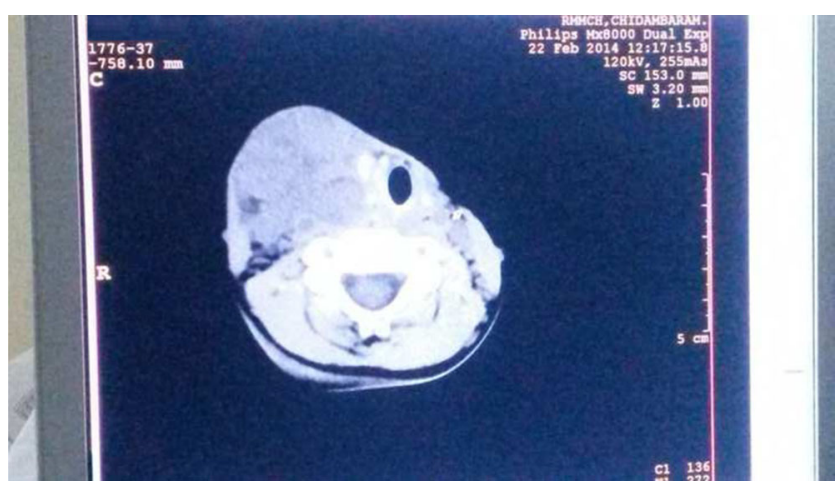

Fig. 1 CT scan neck showing mixed cystic solid lesion in the deep anterior and lateral compartment

gland. Post-operative period was uneventful. Histopathology revealed numerous dilated vascular channels lined by endothelium embedded in fibrocollagenous tissue suggestive of vascular hamartoma. This case is being presented because of the peculiarity of its clinical presentation and to emphasize that vascular malformation should be considered in the differential diagnosis of neck swellings.

\section{Discussion}

Vascular anomalies are congenital lesions of abnormal vascular development commonly referred to as vascular birthmarks. They may occur anywhere in the body common area being skin of the head and neck. Vascular anomalies were classified into haemangiomas and vascular malformations by Mulliken and Glowacki in 1982 based on their histology, biological behaviour and clinical presentation [1].

Vascular malformations are irregular networks of vascular channels caused by localised defect in vascular morphogenesis whereas haemangiomas are true benign vascular neoplasms [2]. They are slow growing, infiltrative and destructive. Developmentally, they are localized or diffuse errors of

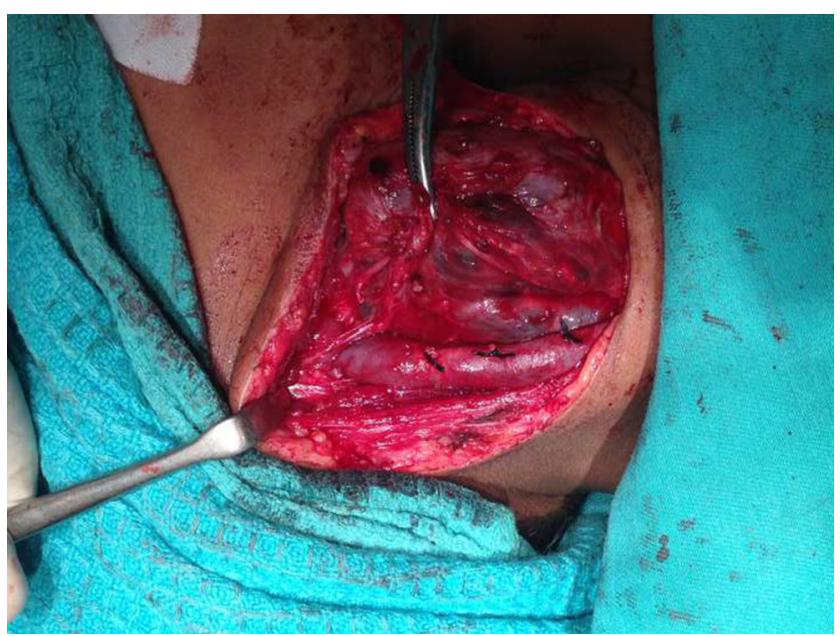

Fig. 2 Perioperative picture showing infiltration to carotid sheath embryonic development characterized by normal endothelial cell turnover, altered vessel architecture. Histologically, vascular malformations are composed of dilated vascular channels lined by flattened, but mature endothelium, and are lined by a thin, unilamellar basement membrane [3].

The clinical manifestation of vascular malformations is extremely variable, depending on the location, the type of malformation and the degree of soft-tissue, muscle and bone involvement. Symptoms range from simple skin discolorations to large devastating deformations. Most of them affect the skin and are therefore often obvious at birth. But some of them may not manifest until childhood or until puberty.

Vascular malformations are subdivided anatomically and rheologically into slow-flow lesions (capillary, venous and lymphatic malformations), fast-flow lesions (arterial and arteriovenous malformations) and complex combined lesions $[4,5]$

Diagnosis of vascular anomalies of the skin and subcutaneous tissue is clinical. Ultrasonogram [6] coupled with colour Doppler study can be used to assess lesion morphology and vessel characteristics. Magnetic resonance imaging plays an important role in diagnosis [7]. It characterizes type of lesion, extent of the lesion, involvement of tissue layers and flow pattern that helps not only in establishing a definite diagnosis, but also helps in guiding treatment.

Management of vascular malformation needs to be individualized based on location, extent of lesion, flow characteristics, accessibility and cosmetic considerations [8]. The main treatment options are surgical excision, embolization, sclerotherapy, laser therapy and cryotherapy. Surgical removal is the treatment of choice in symptomatic well-circumscribed lesions. Sclerotherapy is a simple and feasible alternative to surgery used as a sole modality or in combination with surgery.

\section{Conclusion}

Vascular malformations need to be considered in the differential diagnosis of swellings anywhere in the body. They need to be differentiated from haemangiomas because their treatment is different.

Conflict of Interest The authors have no conflict of interest.

\section{References}

1. Mulliken JB (2013) Vascular malformations of the head and neck. In: Mulliken JB, Burrows PE, Fishman SJ (eds) Vascular anomalies: Hemangiomas and malformations, 2nd edn. Oxford University Press, New York, pp 839-871 
2. Heink KD, Mulliken JB, Kozakewich HP et al (2002) Venous malformations of skeletal muscle. Plast Reconstr Surg 110(7):16251635

3. Chiller KG, Frieden IJ, Arbiser JL (2003) Molecular pathogenesis of vascular anomalies: Classification into three categories based upon clinical and biochemical characteristics. Lymphat Res Biol 1(4):267281

4. Richter GT, Friedman AB (2012) Haemangiomas and vascular malformations: Current theory and management. Int J Paediatr. doi: 10.1155/2012/645678, Accessed online on 22/03/2014 from http:// www.hindawi.com
5. Kubiena H, Madel C. Haemangiomas and vascular malformations from www.meduniwien.ac.at/haemangioma. Accessed on 22 March 2014

6. Paltiel HJ, Burrows PE, Kozakewich PW et al (2000) Soft tissue vascular anomalies: Utility of ultrasound for diagnosis. Radiology 214(3):747-754

7. Fayad LM, Hazirolan T, Dluemke D et al (2006) Vascular malformations in the extremities: Emphasis on MR imaging features that guide treatment options. Skelet Radiol 35(3):127-137

8. Dubois J, Soulez G, Olivia VL et al (2001) Soft tissue venous malformations in adult patients - imaging and therapeutic issues. Radiographics 21(6):1519-1531 